\title{
Ganglioglioma with anaplastic transformation
}

\author{
Ganglioglioma com transformação anaplásica \\ Fabiano Reis ${ }^{1}$, Guilherme Henrique Alves Vieira², Ricardo Schwingel2, Vinicius Trindade Gonçalves², \\ Luciano de Souza Queiroz ${ }^{3}$
}

A nine year-old male had refractory seizures for four years. Diagnostic imaging (Fig 1A and B) demonstrated a cortico-subcortical focal lesion at the isthmus of the right cingulate gyrus. Biopsy yielded diagnosis of ganglioglioma (Fig 2A). Reoperation one year later showed similar features, but included atypical and multinucleated cells (Fig 2B).

At the age of 13, the patient had symptom recurrence. Diagnostic imaging (Fig 1C and D) demonstrated a heterogeneous enhanced lesion in the right parietal region, with necrosis. Histology (Fig 2C to F) revealed a malignant glial tumor with the appearance of glioblastoma multiforme. A few tumor cells were positive for chromogranin. A diagnosis of malignant transformation of ganglioglioma was made.

Gangliogliomas are rare tumors predominating in the early decades of life, with strong association with long term intractable epilepsy ${ }^{1-3}$. They are composed of variable proportions of glial (mainly astrocytic) cells and mature or dysplastic neurons². Malignant change is a rare, but well recognized, complication. Transformation of the glial component from the low grade to a higher grade is observed in most cases. Also, there is a case of malignant transformation secondary to degeneration of the neuronal component into a neuroblastoma ${ }^{4}$. Some reports in literature ${ }^{5}$ suggest that radiation may predispose to malignant degeneration. In this case, the patient did not receive postoperative radiation.
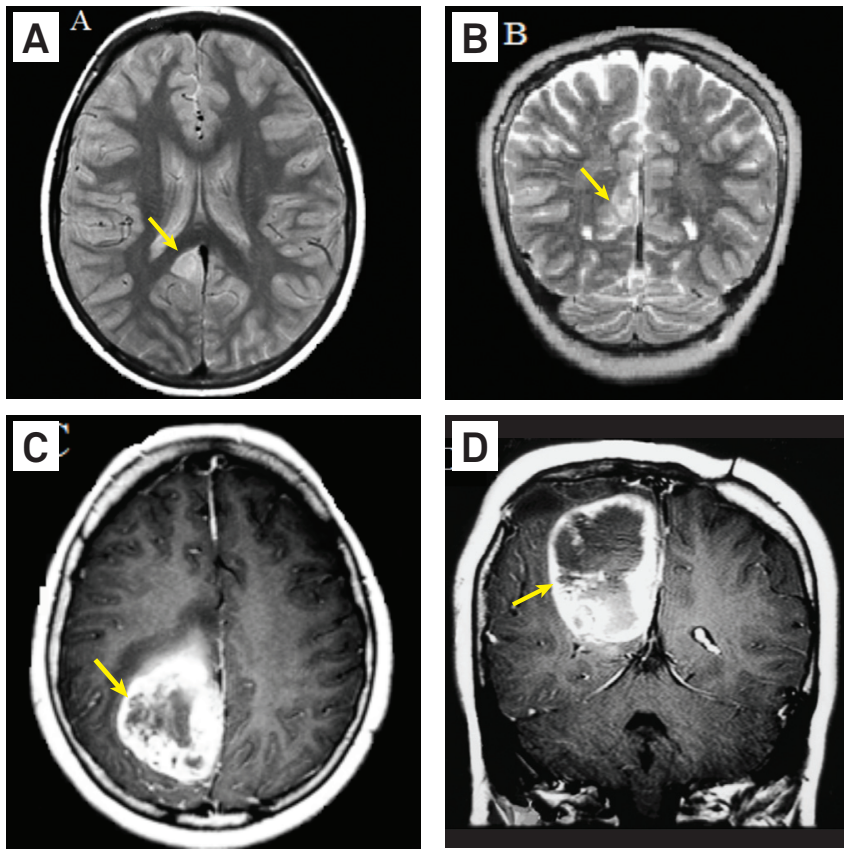

Fig 1. (A) Axial proton density: a small well circumscribed lesion in the right cingulus; (B) Coronal T2: a small lesion in the right cingulus, with high signal intensity in the mass. (C) and (D) Axial and coronal contrast-enhanced, 5 years after: a large mass in the right parietal region, with heterogeneous enhancement and component of necrosis.

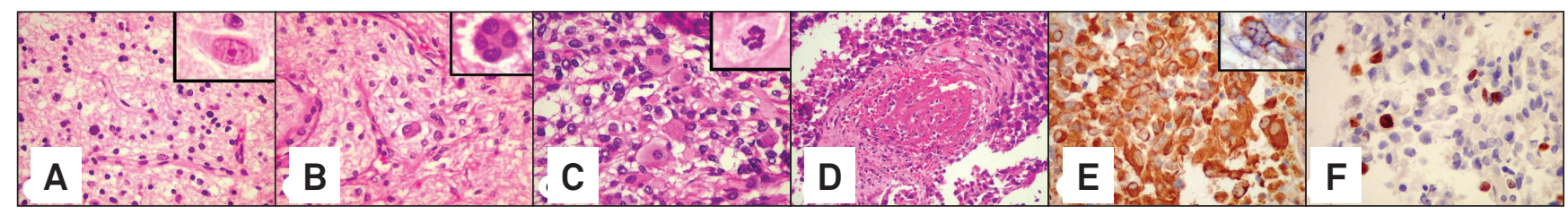

Fig 2. Neuropathology. (A) First biopsy. Moderately cellular low grade glial tumor with scanty atypical cells and thin capillaries. No mitotic figures or necrosis. HEx 100. Inset. Neuron in deep area of the tumor. HEx 400. (B) Second biopsy, one year later. It keeps same features as original specimen. At center, atypical neuron with eccentric nucleus. HEx 100. Inset. Aberrant cell with four nuclei, lineage uncertain. HE x 400. (C-F) Third biopsy. (C) Highly cellular tumor with moderate atypia. HEx 100. Inset. Atypical mitotic figure. HE x 400. (D) Abnormal vessel with thickened walls and occlusive thrombosis. HEx 100. Necrotic areas were present nearby. (E) Tumor cells are strongly positive for glial fibrillary acidic protein indicating astrocytic lineage. X 100 . Inset. Isolated tumor cell positive for chromogranin (suggests neuronal differentiation).X 400. (F) About 10\% of nuclei were marked by Ki-67 (mib1) antibody. X 100.

\footnotetext{
1Professor of the Department of Radiology of the Clinics Hospital, Faculty of Medical Sciences, Universidade Estadual de Campinas (Unicamp), Campinas SP, Brazil. ${ }^{2}$ Medical student; Department of Radiology of the Clinics Hospital, Faculty of Medical Sciences, Unicamp, Campinas SP, Brazil. ${ }^{3}$ Professor of the Department of Pathology of the Clinics Hospital, Faculty of Medical Sciences, Unicamp, Campinas SP, Brazil. Correspondence: Fabiano Reis; Departamento de Radiologia, Faculdade de Ciências Médicas, Unicamp; Rua Tessália Vieira de Camargo 126 ; $13083-887$ Campinas SP - Brasil; E-mail: fabianoreis2@gmail.com

Conflict of interest: There is no conflict of interest to declare.

Received 29 February 2012; Received in final form 17 May 2012; Accepted 25 May 2012
} 


\section{References}

1. Miller DC, Lang FF, Epstein FJ. Central nervous system gangliogliomas. Part I: Pathology. J Neurosurg 1993;79:859-866.

2. Lantos PL, VandenBerg SR, Kleihues P. Tumors of the nervous system. In: Graham DI, Lantos PL (Eds). Greenfield's neuropathology. 6th ed. London, England:Arnold 1997:583-794.

3. Luyken C, Blümcke I, Fimmers R, et al. The spectrum of long-term epilepsy-associated tumors: long-term seizure and tumor outcome and neurosurgical aspects. Epilepsia 2003; 44:822-830.
David KM, de Sanctis S, Lewis PD, Noury AM, Edwards JM. Neuroblastomatous recurrence of ganglioglioma. Case report. J Neurosurg 2000;93:698-700.

5

Rumana CS, Valadka AB. Radiation therapy and malignant degeneration of benign supratentorial gangliogliomas. Neurosurgery 1998;42:1038-1043. 